\title{
Fuzzy and Reflection in the Construction of a Medical Expert System
}

\author{
Boris A. Kobrinskii \\ Federal Research Center "Computer Science and Control” of RAS, Moscow, Russia \\ Email:kba_05@mail.ru
}

How to cite this paper: Kobrinskii, B.A. (2020) Fuzzy and Reflection in the Construction of a Medical Expert System. Journal of Software Engineering and Applications, 13, 15-23.

https://doi.org/10.4236/jsea.2020.132002

Received: January 26, 2020

Accepted: February 26, 2020

Published: February 29, 2020

Copyright (c) 2020 by author(s) and Scientific Research Publishing Inc. This work is licensed under the Creative Commons Attribution International License (CC BY 4.0).

http://creativecommons.org/licenses/by/4.0/

\begin{abstract}
The fuzzy of symptoms (including visual images), representations and assessments in medicine correspond to the peculiarities of the picture of the world of the patient and the physician taking into account the influence of reflection. The continuum of intermediate characteristics of the signs creates serious difficulty for their assessment by physicians. Experts' confidence factors not only for linguistic features, but also for visual images can help increase the hypothesis quality in intelligent medical diagnostic systems.
\end{abstract}

\section{Keywords}

Fuzzy, Confidence Factors, Reflection, Health Intermediate States, Visual Images

\section{Introduction}

The health is characterized by a continuum of fuzzy transitional states, including normal variants, compensated functional changes, borderline conditions (preillness), chronic diseases in the compensation stage and chronic diseases in the decompensation stage [1]. Minor changes in the body systems cannot be detected, but at certain stages they become available for detection by modern methods for investigation. This determines the need for the earliest possible identification and assessment of such "inflection points" on the "curve" of the continuum. Experienced physicians earlier detect changes that allow us to talk about pathological abnormalities in the body. This is determined by the level of knowledge, experience and intuition. Reflection of the physician allows you to more effectively analyze the slightest pathological manifestations. However, the fuzzy of the signs determines the bias in the estimates of the observed phenomena in different physicians.

The ambiguity of assessments in medicine is determined by the following fac- 
tors: the patient's sensations, the observed signs, the physician's reflection in assessing the role of symptoms, difficulty in analyzing images obtained in radiological studies. At the same time, the underlying principle of soft computing is: "tolerance for inaccuracy, uncertainty, and partial truth to achieve ease of manipulation, robustness, low-cost solutions, and better accordance with reality" [2].

In orphan diseases, there are often varieties, uncertainties and inaccuracies in clinical manifestations [3] [4]. This is the case with regard to the detected signs and the appearance of the patients. In addition, the subjectivity of the patient's complaints and the subjective-objective assessment of the physician during examination of the patient, determined by the experience and the physician specialization, form two layers of inaccuracy, uncertainty of data.

The basis of the methods of working with inaccuracy of information, approximate reasoning and calculations with words is fuzzy logic [5]. In the extended representation, fuzzy logic is equivalent to the theory of fuzzy sets, that is, classes with imprecise, fuzzy boundaries, as defined by L. Zadeh [6] .

The article reveals that outside the classical paradigm each linguistic feature can include two certainty factors simultaneously, one on the time of the manifestation and one on the degree of intensity. This approach was used in the creation of the expert system on orphan diseases [7]. It is also necessary for expert evaluations to take into account the elements of reflection. The image rows of fuzzy phenotypic changes that were not previously considered as elements of expert systems can offer additional knowledge about diseases [8]. This work stands apart from other studies in the field of fuzzy logic in intelligent systems, with its integrated approach to the assessment of linguistic features and images that incorporates the reflections of experts.

\section{Fuzzy}

Physicians of different specialties may have their own opinion on some manifestations of the disease. For example, a therapeutist and a reanimator can evaluate the patient's condition differently because there are no extremely severe patients in therapy, and there are no moderate patients and lighter patients in intensive care. Accordingly, physicians of different specialties can group patients differently. In clinical medicine, the assessment of changes in the condition of patients and individual signs in dynamics is of great importance, which corresponding to the concept a time series.

Similarity and difference are considered together as similarity functions and difference functions and under the general name of similarity functions. Similarity and dissimilarity functions are special types of symmetric association function taking values in $[0,1]$. Symmetric and reflexive correlation functions take values in $[-1,1][9]$.

The importance of fuzzy rules is determined by proximity to human intuition [2]. When referring to medical diagnostics, an intuitive sense of the physician is 
important, based on personal experience, including visual memories, and knowledge obtained from various sources. An intuitive-imaged component is of great practical importance for decision-making. Accordingly, expert physicians keep in their memory optic images of similar cases of diseases and linguistic characteristics of atypical cases of pathology, which can serve as a context for visual image representations.

\section{Reflection}

Hypotheses and alternatives to these hypotheses are determined by how a person interprets one or another phenomenon, its psychological setting and intention in the current situation [10]. Constructs, as a subjective tool, include a professional component and personal perception, including reflection. Constructs can be both verbal (conscious constructs that can be expressed in words) and preverbal (unconscious). To measure personality constructs, the methodological principle of "repertory grids" and the repertoire test of personality constructs were developed. In the generalization to the case of multivalued Boolean logic, the choice is made on a partially ordered set of estimates [11].

In medical applications, there is often a fuzzy interpretation of subjective judgments about the plausibility and truth of facts, about the intensity of relations between elements of the semiotic system (degree of similarity, preference), and many fuzzy ideas arise. A different degree of confidence, depending on the nature of the image of the disease arising at the physician, may be a reflection of incomplete information or polymorphism of the clinical picture, i.e. fuzziness, which increases the reflectivity of the decision maker.

As you know, with incomplete, inaccurate and variable information, our reasoning is often conjectural, which makes them in fact only plausible in relation to the true picture of the world. In this regard, a number of hypotheses are constructed with arguments for and against, taking into account the incompleteness and fuzziness of various signs.

The fuzzy of concepts and possible fuzzy in assessing their assignment to a particular class can also periodically change (decrease/increase) due to changes in associative relationships with other signs and the reflexivity of the physician.

Reflection leads to increased attention to certain information (activation of certain images) and to an artificial reduction in the role of other data. It is present at all stages of perception/processing of data and observed or mentally restored visual images.

The physician's scientific preferences inadvertently lead to increased attention in relation to certain information (activation of certain signs and images) and an artificial decrease in the role of other data immersed in the depths of consciousness. At the physician-expert, reflection is manifested in the process of extracting knowledge to create an intelligent system for supporting the adoption of diagnostic or therapeutic decisions. This is especially true for various image representations (situations as images based on the multicomponent nature of signs, 
visual images). The expert's thought process, including reflection, can be implemented in a formalized form and presented on the basis of a formula that includes a fuzzy variable, a confidence factor, the relevance of symptoms and the temporal characteristics of changes in the course of diseases [12].

Correction of the knowledge base during its testing (changing the system of rules) to increase the efficiency of the expert system can be considered as an analogue of the reflexive process in humans.

In [13], general definition of resemblance measures is used as symmetric real valued functions that are reflexive or irreflexive.

\section{Confidence Factors}

Fuzzy and probability, which model different types of uncertainty, are mutually complementary, and a measure of fuzziness of a fuzzy set could also serve as a measure of the uncertainty that arises when deciding which class of objects of an analyzed set belongs to [14]. This implies the importance of the hypothesis being accompanied by an indication of the physician's degree of confidence in her belonging to a particular situation, bearing the existing knowledge about the manifestations of this disease. This information can be used to build hypotheses regarding diseases characterized by a variety of clinical options (masks).

In the case of evaluating a holistic imaginative and at the same time symbolic situation (for example, such concepts as "rough facial features", "elf s face", etc.), we should talk about determining the compatibility function not on the set of mathematically precisely defined objects, but on the set indicated by some symbols of impressions [15]. To process such judgments, a numerical scale of properties is proposed, which are not based on objectively measurable (numerical) values, but rather by an expert on the properties of the observed phenomenon. With such an interpretation of subjective judgments in the form of a set of fuzzy objects, there will be more naturally some linearly ordered set that is different from the interval $[0,1]$, for example, a great number of linguistic evaluations ("sharply expressed", ..., "reminds") presented in decimal or other scale. This corresponds to the idea of the severity of signs of disease.

Knowledge of medical experts is necessary to formation diagnostic systems in the field of orphan diseases, in connection with the extremely low frequency of their occurrence [3] [16], which does not allow the effective use of other approaches. In addition, one should take into account the polyvariance and the severity of temporary changes in primary and secondary signs, characterized by various peculiarities of manifestations.

Difficultly recognizable visual radiological biomarkers (image biomarker) can be qualitative, which also requires expert interpretation for the purpose of highthroughput quantitative image analysis [17].

At the same time, in the process of formation fuzzy models, there are two contradictory requirements: the model must reproduce the system under investigation (accuracy) as accurately as possible and must express the behavior of the 
system in a form understandable to humans (interpretability). It is impossible to build a model at the same time with a high degree of accuracy and interpretability, because these properties are contradictory in practical terms, one of them prevails over the other and the result can be the achievement of only varying degrees of interpretability and accuracy [18]. Conditional accuracy can be adjusted by expert opinions expressed by factors of their confidence in the accuracy of manifestation and detection of signs in accordance with the traditional approach proposed by E.H. Shortliffe and B.G. Buchanan [19] in the Stanford model. Confidence measures or certainty factors determining the expert's views on the significance of signs are intuitive-interval. At the same time, an intuitive interpretation allows to interpret the situation for different variants of the disease. At the same time, the granular mathematics of Zadeh [20], being an interval, creates opportunities for assessing dynamically changing states, an increase in pathological manifestations, which is especially important in diseases, accompanied by the accumulation of macromolecules in various organs.

All elements within the boundary region can have a conditional place, because they only partially satisfy the conditions for getting into an unbounded region of the set [21]. While the conditions would be completely fulfilled, these elements would be unconditional members of a particular set. As described in [22], opportunity is considered as a measure of the degree to which the condition is fulfilled.

At the same time, the factors of confidence associated with conditional probabilities, but not similar to them. On the one hand, E. Rich et al. [23] admit that reliability factors smaller than zero represent cases where some evidence tends to disprove some hypothesis. At the same time, the authors discuss that the reliability (confidence) factors consist of two components: measures of faith and measures of unbelief.

Confidence measures or the factors of confidence exhibited by experts often cannot belong to the whole class (subclass), but relate to individual groups (subgroups). This is especially pronounced in medicine, in particular in various age groups and in the dynamics of diseases. That is, the confidence factors of symptoms are associated with signs of the clinical picture of the disease in various periods.

For example, the experts agreed on three confidence factors for the sign of hepatomegaly: manifestation of the sign at a certain age at 0.6 , frequency of occurrence at 0.4 , and severity at 0.3 . Confidence factors in the next age range increase to $0.6,0.5$, and 0.4 respectively [7].

In the trend of chronic diseases, there is a fuzzy in assessing the dynamics of symptoms. The transformation of symptoms in the course of the disease, the depth of knowledge of physician-experts and their subjective preferences determine the assessment of confidence factors. Especially difficult is the assessment of traits, which are boundary elements that define fuzzy transitions from one age group to another, from one period of the disease to another. In this regard, cer- 
tainty factors were proposed for the manifestation and severity of each symptom [7], which are a condition for increasing the reliability of solutions in expert systems.

Formed granules can be accompanied by certainty factors $[-1,+1]$ and regarding the similarity with a typical visual image (archetype), if we consider visual images as signs.

In clinical terms, variants of high similarity of granules are possible and, conversely, symptoms of the disease in the form of different individual small granules, due to the polymorphism of clinical manifestations and the fuzzyness of individual signs characterized by large or smaller differences on the linguistic scale, on the scale of order, on metric scales.

Fuzzy clustering based on frequency prototypes and dissimilarity measures is possible for categorical data arrays [24].

\section{Fuzzy Image Rows}

Consider the option of fuzzy constraints in concepts formed on the basis of holistic images in an iconic picture of the world.

Let's turn to the concept of fuzzy relation by Lotfi Zadeh [6] for consideration of similarity measures as functions in universal domain satisfied some sets of properties.

Computer-aided image analysis is becoming increasingly important to efficiently handle high-resolution images generated by advanced medical devices for visualization [25].

Sequence of similar manifestations of the visual image corresponding to one notion can be considered as a fuzzy set or an image row [8] (Figure 1).

Differences in individual images will be determined not only by manifestations of pathology, but also by ethnic characteristics of individuals.

The presentation of a previously unknown similar representative of such a series allows us to identify this image, combining it with a known multitude. It is clear that in the human memory either typical representatives of each subgroup (possibly subjected to simplification) or a set of images reflecting the specific features of the original symbol are stored. On this basis, a figurative row can be built in the knowledge base of the expert system.

As proanalogues of images, which are symbols, one can consider archetypes or images-prototypes as indicators of concrete image rows. Characteristic medical signs can be analyzed based on a study of their contours [26].
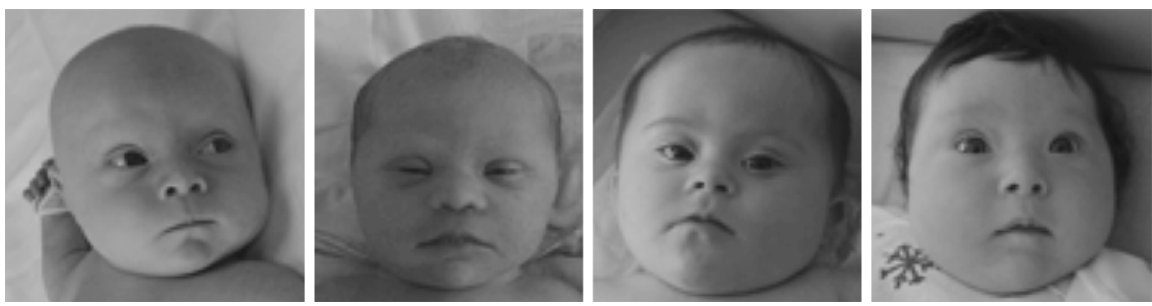

Figure 1. Faces of patients with Down's syndrome. 
Image rows of phenotypic manifestations of the disease can serve as the basis for visual diagnosis of diseases. The images of patients of one visual series, having certain differences from each other, are a conditionally fuzzy image of a representative of a certain pathological condition. Distinction between representatives of image rows is the basis for differential diagnosis.

The temporal dynamics of the pathological process, especially with progressively current diseases, is a set of images in row, having similar features.

The inclusion in intelligent systems of image rows can allow you to solve problems of finding characteristic unifying features among images of the same type (for example, the faces of patients with a single disease).

The concept of an image row can be perceived as quasi-continuum of close or relatively close images, which implies the fuzzy of transitions between individual representatives of row. But a tuple of patients similar in appearance forms an image row of one subset, and sometimes of one set. However, the appearance and individual external signs of patients can be both similar and difficult to distinguish in various diseases.

Therefore, for holistic visual images, such as a scaphoid skull, a sphinx face, a fish mouth, etc.), it is advisable to use expert confidence factors.

\section{Conclusions}

Many chronic diseases progress with age, which leads to the appearance of new symptoms and an increase in the severity of previously manifested signs. This process is continuous. The fuzzy of the clinical picture is manifested in a more pronounced form in hereditary diseases.

The effectiveness of diagnostic hypotheses in expert systems is possible based on a combination of confidence factors verbalized signs and visual holistic images, the period of manifestation and the severity of symptoms in a certain period of life (in particular for various ethnic groups). These evaluations show the reflexivity of the expert, which should be taken into account by the cognitologist.

\section{Conflicts of Interest}

The authors declare no conflicts of interest regarding the publication of this paper.

\section{References}

[1] Kobrinsky, B. (1995) Concept of the Continuum of Intermediate States of Development: Risk Factors in Child Health. Medical Audit News, 5, 21-22.

[2] Zadeh, L.A. (1993) The Role of Fuzzy Logic and Soft Computing in the Conception and Design of Intelligent Systems. In: Klement, E.P. and Slany, W., Eds., Fuzzy Logic in Artificial Intelligence, FLAI 1993, Lecture Notes in Computer Science (Lecture Notes in Artificial Intelligence), Springer, Berlin, Heidelberg, Vol. 695, 1. https://link.springer.com/chapter/10.1007/3-540-56920-0_1 https://doi.org/10.1007/3-540-56920-0_1

[3] Aronson, J. (2006) Rare Diseases, Orphan Drugs, and Orphan Diseases. British Medi- 
cal Journal, 333, 127-127. https://doi.org/10.1136/bmj.333.7559.127

https://www.researchgate.net/publication/274431884_Rare_diseases_orphan_drugs _and_orphan_diseases

[4] Bavisetty, S., Grody, W.W. and Yazdani, S. (2013) Emergence of Pediatric Rare Diseases: Review of Present Policies and Opportunities for Improvement. Rare Diseases, 1, e23579. https://www.ncbi.nlm.nih.gov/pubmed/25002987 https://doi.org/10.4161/rdis.23579

[5] Malik, D.S. and Mordeson, J.N. (2000) Fuzzy Discrete Structures. Studies in Fuzziness and Soft Computing, Vol. 58, Physica-Verlag, Heidelberg. https://www.springer.com/gp/book/9783790813357 https://doi.org/10.1007/978-3-7908-1838-3

[6] Zadeh, L.A. (1971) Toward a Theory of Fuzzy Systems. In: Kalman, R.E. and Claris de, N., Eds., Aspects of Network and System Theory, Holt, Rinehart and Winston, New York, 469-490.

[7] Kobrinskii, B.A., Demikova, N.S. and Blagosklonov, N.A. (2018) Knowledge Engineering in the Expert Systems Construction on Hereditary Diseases. Communications in Computer and Information Science Series, Vol. 934, RCAI: Advances in Artificial Intelligence and Applications, Proceedings of the 16th Russian Conference on Artificial Intelligence, Moscow, 24-26 September 2018, 35-45.

https://link.springer.com/chapter/10.1007/978-3-030-00617-4_4

https://doi.org/10.1007/978-3-030-00617-4_4

[8] Kobrinskii, B.A. (2013) The Significance of Visual_Image Presentations for Medical Intelligent Systems. Scientific and Technical Information Processing, 40, 337-341. https://link.springer.com/article/10.3103/S014768821306004X https://doi.org/10.3103/S014768821306004X

[9] Batyrshin, I. (2019) Towards a General Theory of Similarity and Association Measures: Similarity, Dissimilarity and Correlation Functions. Journal of Intelligent and Fuzzy Systems, 36, 2977-3004. https://doi.org/10.3233/JIFS-181503

https://content.iospress.com/articles/journal-of-intelligent-and-fuzzy-systems/ifs18 $\underline{1503}$

[10] Kelly, G.A. (1963) A Theory of Personality: The Psychology of Personal Constructs. W.W. Norton and Company, New York.

[11] Taran, T.A. (2001) Many-Valued Boolean Model of the Reflexive Agent. Journal Multiple Valued Logic, 7, 97-127.

[12] Kobrinskii, B.A. (2017) Expert Reflection in the Process of Diagnosis of Diseases at the Extraction of Knowledge. Advances in Computer Science Research, Vol. 72, Proceedings of the IV International Research Conference "Information Technologies in Science, Management, Social Sphere and Medicine", Russia, Tomsk, December 5-8, 2017, 321-323.

https://www.atlantis-press.com/proceedings/itsmssm-17/25887897 https://doi.org/10.2991/itsmssm-17.2017.66

[13] Batagelj, V. and Bren, M. (1995) Comparing Resemblance Measures. Journal of Classification, 12, 73-90. https://doi.org/10.1007/BF01202268 https://link.springer.com/article/10.1007/BF01202268

[14] Zadeh, L.A. (1995) Discussion: Probability Theory and Fuzzy Logic are Complementary Rather than Competitive. Technometrics, 37, 271-276. https://doi.org/10.1080/00401706.1995.10484330

[15] Batyrshin, I.Z. (1996) Methods for Presenting and Processing Fuzzy Information in Intelligent Systems. Artificial Intelligence News, 2, 59-65. 
[16] Richter, T., Nestler-Parr, S., Babela, R., et al. (2015) Rare Diseases Terminology and Definitions: A Systematic Global Review: Report of the ISPOR Rare Disease Special Interest Group. Value Health, 18, 906-914.

https://www.ncbi.nlm.nih.gov/pubmed/26409619 https://doi.org/10.1016/j.jval.2015.05.008

[17] Zwanenburg, A., Leger, S., Vallieres, M. and Lock, S. (2019) Image Biomarker Standardisation Initiative. https://arxiv.org/pdf/1612.07003.pdf

[18] Gasto, M.J., Alcala, R. and Herrera, F. (2011) Interpretability of Linguistic Fuzzy Rule-Based Systems: An Overview of Interpretability Measures. Information Sciences, 181, 4340-4360. https://sci2s.ugr.es/fuzzyInterpretability https://doi.org/10.1016/j.ins.2011.02.021

[19] Shortliffe, E.H. and Buchanan, B.G. (1984) A Model of Inexact Reasoning in Medicine. In: Buchanan, B.G. and Shortliffe, E.H., Eds., Rule-Based Expert Systems. The MYCIN Experiments of the Stanford Heuristic Programming Project, Addison-Wesley Publ. Co., London, Amsterdam, Sydney, 233-262.

http://people.dbmi.columbia.edu/ ehs7001/Buchanan-Shortliffe-1984/Chapter-11.p df

[20] Zadeh, L.A. (1997) Towards a Theory of Fuzzy Information Granulation and Its Centrality in Human Reasoning and Fuzzy Logic. Fuzzy Sets and Systems, 90, 111-127. https://www.sciencedirect.com/science/article/abs/pii/S0165011497000778 https://doi.org/10.1016/S0165-0114(97)00077-8

[21] Liu, H. and Cocea, M. (2017) Fuzzy Information Granulation towards Interpretable Sentiment Analysis. Granular Computing, 2, 289-302. https://link.springer.com/article/10.1007/s41066-017-0043-8 https://doi.org/10.1007/s41066-017-0043-8

[22] Liu, H., Gegov, A. and Cocea, M. (2016) Rule Based Systems: A Granular Computing Perspective. Granular Computing, 1, 259-274.

https:/link.springer.com/article/10.1007\%2Fs41066-016-0021-6 https://doi.org/10.1007/s41066-016-0021-6

[23] Rich, E., Knight, K. and Nair, S.B. (2008) Artificial Intelligence. Third Edition, Tata McGraw-Hill Publ. Co. Ltd., New Delhi.

https://www.abebooks.de/Artificial-Intelligence-Third-Edition-Elaine-Rich/501298 $\underline{3601 / \mathrm{bd}}$

[24] Hu, Zh., Bodyanskiy, Y.V., Tyshchenko, O.K. and Samitova, V.O. (2017) Possibilistic Fuzzy Clustering for Categorical Data Arrays Based on Frequency Prototypes and Dissimilarity Measures. International Journal of Intelligent Systems and Applications, 9, 55-61. http://www.mecs-press.org/ijisa/ijisa-v9-n5/IJISA-V9-N5-7.pdf https://doi.org/10.5815/ijisa.2017.05.07

[25] Olabarriaga, S.D., Snel, J.G., Botha, C.P. and Belleman, R.G. (2007) Integrated Support for Medical Image Analysis Methods: From Development to Clinical Application. IEEE Transactions on Information Technology in Biomedicine, 11, 47-57. http://visualization.tudelft.nl/Publications-new/2007/OSBB07a/OSBB07a.pdf https://doi.org/10.1109/TITB.2006.874929

[26] Zewail, R. and Hag-ElSafi, A. (2017) Appearance-Based Salient Features Extraction in Medical Images Using Sparse Contourlet-Based Representation. International Journal of Image, Graphics and Signal Processing, 9, 1-10. http://www.mecs-press.org/ijigsp/ijigsp-v9-n9/IJIGSP-V9-N9-1.pdf https://doi.org/10.5815/ijigsp.2017.09.01 